\title{
Overexpectation in appetitive Pavlovian and instrumental conditioning
}

\author{
K. MATTHEW LATTAL and SADAHIKO NAKAJMA \\ University of Pennsylvania, Philadelphia, Pennsylvania
}

\begin{abstract}
In three experiments that used appetitive preparations with rats, we examined the effects of reinforcing a compound consisting of two previously reinforced stimuli on subsequent responding to those stimuli. Experiment 1 showed that a Pavlovian conditioned stimulus given this treatment evoked fewer magazine entries when presented alone than did a reinforced stimulus that did not receive the compound treatment. Experiment 2 examined inhibition of delay and generalization decrement accounts for the results of Experiment 1. Experiment 3 extended this finding to an instrumental learning paradigm. These results demonstrate overexpectation in appetitive Pavlovian and instrumental paradigms. The implications for current theories of conditioning are discussed.
\end{abstract}

Many theories of Pavlovian and instrumental conditioning incorporate the assumption that the effects of reinforcement depend on the discrepancy between the reinforcer received and that anticipated (see, e.g., Dickinson, 1980, 1989; Kamin, 1969; Pearce, 1987; Rescorla \& Wagner, 1972; Wagner, 1981). The same reinforcer can have a large or a small incremental effect, depending on the degree to which it is anticipated. Indeed, according to many of these theories, that same reinforcer can have a decremental effect if it is weaker than was anticipated. The adoption of this assumption has been widespread, not only in the context of conditioning, but also in related work on human causality judgments (e.g., Chapman \& Robbins, 1990; Dickinson, Shanks, \& Evenden, 1984), and is inherent in the learning rules adopted in most connectionist models (e.g., Kehoe, 1988; Shanks, 1991). Despite the theoretical ubiquity of such an assumption, there are few clear demonstrations of the decremental effects of reinforcement on associative learning.

One prominent early theory that makes this assumption is the Rescorla-Wagner model (Rescorla \& Wagner, 1972). This theory characterizes anticipated and obtained reinforcers in terms of the current associative strength $(V)$ of a conditioned stimulus (CS) and the total associative strength that an unconditioned stimulus (US) supports $(\lambda)$. Changes

This research was supported by National Science Foundation Grant IBN94-04676 to Robert A. Rescorla. S.N. was supported by the Japan Society for Promotion of Science's Postdoctoral Fellowship for Research Abroad. He is now at the Department of Psychology, Kwansei Gakuin University, Nishinomiya, Japan. We thank Robert A. Rescorla for many helpful discussions and for comments on earlier versions of the manuscript. We also thank Juan M. Rosas-Santos for comments on an earlier version of the manuscript. Correspondence concerning this article should be addressed to K. M. Lattal, Department of Psychology, University of Pennsylvania, 3815 Walnut Street, Philadelphia, PA 19104 (e-mail: lattal@cattell.psych.upenn.edu).

-Accepted by previous editor, Robert A. Rescorla in associative strength occur until there is no discrepancy between the anticipated and obtained reinforcers (i.e., until $V_{\mathrm{CS}}=\lambda$ ). When two CSs (e.g., A and X) are presented in compound, the total associative strength of the compound equals the sum of the component stimuli $\left(V_{\mathrm{AX}}=V_{\mathrm{A}}+V_{\mathrm{X}}\right)$. In this case, each stimulus element of the compound changes as a function of the discrepancy between $V_{\mathrm{AX}}$ and $\lambda$. The associative strengths $V_{\mathrm{A}}$ and $V_{\mathrm{X}}$ increase, decrease, or remain the same, depending on whether $V_{\mathrm{AX}}$ is less than, greater than, or equal to $\lambda$. The magnitude of the change in $V_{\mathrm{A}}$ and $V_{\mathrm{X}}$ depends on the learning rate parameters associated with each (see, e.g., Kamin \& Gaioni, 1974).

A major success of this model lies in its ability to account for blocking (Kamin, 1968). In this paradigm, a stimulus (A) is reinforced to asymptote $\left(V_{\mathrm{A}}=\lambda\right)$ and subsequently is reinforced in compound with a neutral stimulus (X), which has no associative strength. Because $V_{\mathrm{AX}}=$ $V_{\mathrm{A}}+V_{\mathrm{X}}$, the total associative strength of the compound is equal to that of $\mathrm{A}$ alone (i.e., $V_{\mathrm{AX}}=\lambda$ ). Reinforcing the compound does not lead to any change in the associative strengths of the elements, because there is no discrepancy between $V_{\mathrm{AX}}$ and $\lambda$. Stimulus $\mathrm{X}$ therefore gains no associative strength. However, if $\mathrm{X}$ had been reinforced independently in a manner similar to that for A, reinforcing the stimuli in compound leads the model to a surprisingly different prediction - each element should decrease in associative strength, because $V_{\mathrm{AX}}$ is larger than $\lambda$. Continued $\mathrm{AX}$ reinforcement will decrease $V_{\mathrm{A}}$ and $V_{\mathrm{X}}$ until their sum equals $\lambda$-that is, until the anticipated and obtained reinforcers are of the same magnitude.

The introduction of the Rescorla-Wagner model was accompanied by some early demonstrations of such an overexpectation effect (Kremer, 1978; Levitan, 1975; Rescorla, 1970; see also Kamin \& Gaioni, 1974; Wagner, 1971). For example, Rescorla (1970) found that separate reinforcement of two stimuli ( $\mathrm{A}$ and $\mathrm{X}$ ) followed by reinforcement of the AX compound caused a decrease in responding to $\mathrm{X}$ at a test, relative to a group that did not re- 
ceive the compound treatment. This group difference was offered as evidence of overexpectation.

This was a surprising and influential finding. As much as any other result, it has supported the almost universally adopted assumption that the effect of a reinforcer is not fixed but depends on its relation to the current expectations of the organism. Indeed, the theoretical importance of overexpectation rivals that of blocking, but, although there have been hundreds of demonstrations of blocking, there have been only a handful of experimental reports of overexpectation. Moreover, virtually all of the successful reports have been conducted with a particular preparationconditioned suppression resulting from the pairing of a CS with shock. As a result, the actual data base on which this important theoretical assumption rests is quite narrow.

The present experiments focus primarily on two points. The first is the possibility of obtaining the effect with an appetitive reinforcer. Although there have been several attempts to generate overexpectation in appetitive preparations, the recent evidence from these studies is quite mixed (Bourne, 1990; Ganesan \& Pearce, 1988; Khallad \& Moore, 1996; Rescorla, 1989). The second is the need for additional controls in order to ensure that the observed decrease is indeed attributable to the compound's evoking greater associative strength than that supported by the US. In particular, the present experiments ensured that the effect could not be attributed to the passage of time, habituation to the US, inhibition of delay in the CS, or the simple reinforcement of an element in compound regardless of the total strength of that compound. These confounding variables were not fully controlled in previous studies that addressed overexpectation.

A well-controlled demonstration of overexpectation also has important implications for some other modern theories of conditioning. Pearce's $(1987,1994)$ stimulus generalization model is similar to the Rescorla-Wagner model in that it relies on the basic assumption that the discrepancy between obtained and anticipated reinforcers determines the effectiveness of that reinforcer. It differs from the Rescorla-Wagner model by viewing stimuli as configural units, with each unique configuration entering into associations with the reinforcer. Several recent studies have found that the Pearce configural model is superior to the Rescorla-Wagner model in a variety of situations (e.g., Nakajima, 1997; Pearce \& Redhead, 1993, 1995; Wilson \& Pearce, 1992). However, one classic phenomenon that particularly raises problems for the Pearce model is summation-the observation that two separately conditioned stimuli evoke a greater response when presented in compound than they do by themselves (e.g., Kehoe, 1986; Rescorla, 1997). Summation is at the heart of overexpectation; the associative strengths of two stimuli together must be greater than that supported by the US for overexpectation to occur. Because the Pearce model does not routinely predict summation, it also fails to anticipate that overexpectation will be found. The issue of the conditions under which a modified Pearce theory might anticipate summation and overexpectation is addressed in the General Discussion.

A demonstration of overexpectation also is relevant to some recent claims about the ability of biologically significant stimuli to undergo decrements in performance. The comparator theory described by Miller and his colleagues (e.g., Miller \& Schachtman, 1985) anticipates that the performance to a CS will be less, the greater the associative strength of the other stimuli present on a conditioning trial. Such a theory might anticipate overexpectation, because reinforcing a previously trained $\mathrm{A}$ in compound with $\mathrm{X}$ would lead to A's becoming a comparator stimulus for X. Although compound reinforcement would not, on such a theory, reduce the associative strength of $\mathrm{X}$, it would reduce performance to it by modifying its comparator stimulus. However, the available data suggest that simply inflating the value of a comparator stimulus does not adversely affect the response to a previously trained stimulus (Miller, Hallam, \& Grahame, 1990). Recent modifications of the comparator theory intended to incorporate this finding (Denniston, Miller, \& Matute, 1996; Miller \& Matute, 1996) have argued that biologically significant stimuli, such as those previously paired with a US, are not subject to retroactive depression of this sort. As a result, such a modified theory does not anticipate overexpectation.

In the first two experiments reported here, we attempted to demonstrate overexpectation in magazine approach conditioning. In this paradigm, a diffuse stimulus is presented, followed by the delivery of a food pellet in the magazine. Conditioning is assessed by comparing the rates of entry into the magazine, both during the stimulus and during an equal period preceding the stimulus. In the third experiment, we attempted to extend the generality of overexpectation by demonstrating it in an instrumental learning paradigm. Although there is strong evidence that other forms of cue competition, such as blocking and overshadowing, are general to instrumental learning (see, e.g., Williams, 1994), the few prior demonstrations of overexpectation have been limited exclusively to Pavlovian preparations.

\section{EXPERIMENT 1}

The design of this experiment is presented in Table 1. Initially, two stimuli ( $\mathrm{X}$ and $\mathrm{Y}$ ) each were reinforced separately; then, $X$ received additional conditioning when presented in compound with another reinforced stimulus (A). Finally, responding during $X$ and responding during $Y$ were compared in a test. Two results were expected, on the basis of previous research. First, the initial trials of AX compound training should reveal summation-- greater responding during the compound than during the $\mathrm{A}$ and $\mathrm{X}$ elements. Second, $X$ should evoke less responding than should $\mathrm{Y}$ after compound training, thus demonstrating overexpectation. 
Table 1

Experimental Designs

\begin{tabular}{|c|c|c|c|}
\hline \multirow[b]{2}{*}{ Experiment } & \multicolumn{2}{|c|}{ Training } & \multirow[b]{2}{*}{ Testing } \\
\hline & Element & Compound & \\
\hline 1 & $\begin{array}{l}\text { A+ } \\
\mathrm{X}+ \\
\mathrm{Y}+\end{array}$ & $\mathrm{AX}^{+}$ & $\begin{array}{l}\mathrm{X}- \\
\mathrm{Y}-\end{array}$ \\
\hline 2 and 3 & $\begin{array}{l}\mathrm{A}+ \\
\mathrm{B}- \\
\mathrm{X}+ \\
\mathrm{Y}+\end{array}$ & $\begin{array}{l}\mathrm{AX}+ \\
\mathbf{B Y}+\end{array}$ & $\begin{array}{l}\mathrm{X}- \\
\mathrm{Y}-\end{array}$ \\
\hline
\end{tabular}

\section{Method}

Subjects. The subjects were 16 experimentally naive SpragueDawley rats, approximately 90 days old. They were housed in individual cages, where they had free access to water. Supplemental feedings, given $1 \mathrm{~h}$ after the end of each session, maintained the animals at $80 \%$ of their ad-lib body weight.

Apparatus. Four identical operant chambers, measuring $22.9 \times$ $20.3 \times 20.3 \mathrm{~cm}$, were enclosed in sound- and light-attenuating shells. The front and back walls of each chamber were aluminum; the sidewalls and ceiling were clear Plexiglas; the floor was composed of $0.48-\mathrm{cm}$ stainless steel rods, spaced $1.9 \mathrm{~cm}$ apart. A recessed food magazine, measuring $30 \times 36 \times 20 \mathrm{~mm}$, was centered on one wall, $10 \mathrm{~mm}$ above the floor. A pellet dispenser mounted behind the food magazine dispensed 45-mg pellets ( + ; P. J. Noyes Co., Formula A) through a piece of tubing that connected the dispenser to the magazine. Photocells on the inside of the magazine detected head entries. A metal shield covered a lever that was located to the left of the magazine to make it inaccessible. Mounted on the inside wall of each shell was a speaker that could produce a $79-84 \mathrm{~dB}$ re $20 \mu \mathrm{N} / \mathrm{m}^{2}$ white noise and an $1800-\mathrm{Hz}$ tone. The background noise level in the chambers ranged from 70 to $75 \mathrm{~dB}$. A 6-W light bulb also was mounted on the inside wall of each shell and provided steady diffuse light when illuminated. The chamber was dark, except during presentations of the light. Experimental events were controlled and recorded by relays and microprocessors in an adjacent room.

Procedure. During the first session, the animals were trained to enter the food magazine. Food pellets were delivered 20 times, separated by variable intervals that averaged $60 \mathrm{sec}$.

Element training consisted of eight consecutive sessions. The animals were presented with three stimuli, each lasting $30 \mathrm{sec}$. A food pellet was delivered concurrently with the termination of each stimulus. The $\mathrm{X}$ and $\mathrm{Y}$ stimuli were either the tone or the noise, and the A stimulus was the light for all subjects. During Sessions $1-7$, the animals received 12 trials each of $\mathrm{A}+, \mathrm{X}+$, and $\mathrm{Y}+$. The sequence of trials varied, with the constraint that no more than 2 trials of a given stimulus occurred successively. Each trial was separated by an intertrial interval (ITI) that was variable around a mean of $120 \mathrm{sec}$. This ITI was used throughout the experiment.

Prior to Session 8, animals were matched to equate response levels during $X$ and $Y$, while ensuring that the identities of $X$ and $Y$ were distributed equally between the tone and the noise. During the first half of Session 8, element training continued, with 4 trials each of $\mathrm{A}+, \mathrm{X}+$, and $\mathrm{Y}+$. The positions of the stimuli varied across animals, and no stimulus was presented on more than 2 consecutive trials. During the second half of Session 8 , compound training began with 12 trials of concurrent $\mathrm{A}$ and $\mathrm{X}$ presentation, followed by a food pellet $(\mathrm{AX}+)$. Session 9 began with 12 more trials of $\mathrm{AX}+$, followed by 4 nonreinforced test trials each of $X$ and $Y$. For half of the animals, the test sequence was XYYXXYYX; for the other half, it was YXXYYXXY.

In all three experiments, the statistical analyses were performed on CS elevations that were calculated by subtracting the rate of responding during the $30 \mathrm{sec}$ that immediately preceded the CS (the pre period) from the rate of responding during the CS. The statistical test used throughout these experiments was a Wilcoxon matchedpairs $T$ test.

\section{Results and Discussion}

By the end of element training, all animals had acquired the response during $\mathrm{A}, \mathrm{X}$, and $\mathrm{Y}$. During the last four trials of element training, the mean response rates during the pre periods and during $\mathrm{A}, \mathrm{X}$, and $\mathrm{Y}$ were $6.1,15.4,18.9$, and 18.5 responses per minute (rpm), respectively.

During the first trial of compound training, the mean response rates during the pre period and during the $\mathrm{AX}$ compound were 1.8 and $17.6 \mathrm{rpm}$, respectively. The elevation produced by $\mathrm{AX}$ on the first compound trial was reliably larger than the mean of the elevations produced by $\mathrm{A}$ and $\mathrm{X}$ during the final four element-training trials $[T(15)=18, p<.02]$. Some authors have argued that a more conservative measure of comparing the compound to the highest of the elements provides a better assessment of summation (e.g., Aydin \& Pearce, 1997; Rescorla, 1997). This measure-comparing the elevation produced by AX on the first trial with that of the larger of the two component elements-fell short of reliability at conventional alpha levels $[T(14)=24.5, .05<p<.10]$. One factor that complicates a clear description of this summation data is that the pre rates differed from the last four trials of element training to the first trial of compound training $[T(16)=7, p<.01]$. Any summation effect that was evident during the first trial disappeared quickly. During the first four trials of compound training, the mean response rates during the pre period and during the $\mathrm{AX}$ compound were 4.2 and $18.2 \mathrm{rpm}$, respectively. The difference between the elevation produced by $\mathrm{AX}$ on these trials and the mean of the elevations produced by $A$ and $\mathrm{X}$ during the final four element trials failed to reach reliability at the .05 alpha level $[T(16)=30.5, p<.06]$.

Response rates during the compound remained stable over the course of compound training. During the final two trials of compound training, the mean response rates during the pre period and during $A X$ were 4.9 and $16.0 \mathrm{rpm}$, respectively.

Figure 1 shows test responding during $\mathrm{X}$ and $\mathrm{Y}$ in $\mathrm{Ex}$ periment 1 . There was reliably greater elevation during $\mathrm{Y}$ than during $\mathrm{X}$ on the first $[T(16)=23, p<.02]$ and second $[T(16)=25.5, p<.05]$ test trials. By the third trial, there was no reliable difference. In addition, there was no reliable change in responding during $Y$ from the final two training trials to the first two test trials, but there was a reliable decrement in responding during $X[T(16)=19$, $p<.01]$. This change in $\mathrm{X}$ from training to testing was reliably greater than that in $\mathrm{Y}[T(16)=29.5, p<.05]$.

This experiment showed that reinforcement of an $\mathrm{AX}$ compound led to a decrement in the strength of $X$, relative to that of $\mathrm{Y}$, which was spared the compound treatment. This finding replicates that of Rescorla (1970), who used a between-subjects design and an aversive US.

A natural interpretation of this result and of those like it is that reinforced compound presentations of AX led to 


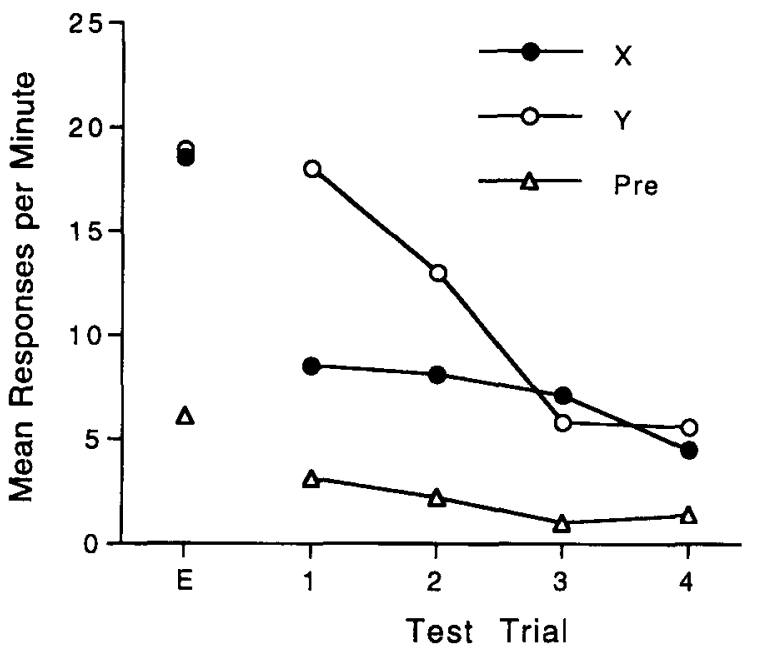

Figure 1. The results of element training and testing in Experiment 1 . The mean response rates are shown during the final four trials of element training (E) and during each test trial for $X, Y$, and the pre period.

a reduction in the strength of $X$. This may have occurred because the excitatory conditioning of $\mathrm{A}$ and $\mathrm{X}$ led the $\mathrm{AX}$ compound to predict a substantially larger reinforcer than that which was received. Because only the original reinforcer was received, the associative strength of $\mathrm{X}$ had to decrease until the predicted reinforcer was equal to the obtained reinforcer, which resulted in a decrease in the associative strength of $X$.

The present experiment rules out one alternative interpretation of overexpectation in terms of habituation to the US. In a between-subjects design, a comparison sometimes is made between a group that receives additional reinforcement of the AX compound and another group that does not. This difference in amount of exposure to the US could be responsible for the observed group differences. However, in a within-subjects design, such as that employed here, any effect of US habituation would apply to both $\mathrm{X}$ and $\mathrm{Y}$. Thus, this experiment eliminates differential US habituation as the basis for an account of the group differences, but two alternative interpretations of the effects of reinforcing the compound are left open by Experiment 1 .

First, one could fashion an account in terms of the inhibition of delay with repeated reinforcement. With the relatively long, 30-sec stimuli used in this experiment, it is common, with extended training, for responding to show a temporal pattern in which it is greater near the end of the stimulus. For instance, a recent magazine approach experiment yielded substantial inhibition of delay in a procedure that was very similar to that in Experiment 1 (Rescorla, 1997). This temporal placement of responding results from a decrease in responding early to the stimulus with additional training. This, in turn, means that the overall response level of a stimulus can be expected to decrease simply as a result of additional training. Thus, in Experiment 1, the continued training of $\mathrm{X}$ during the com- pound phase might have resulted in its developing more inhibition of delay, which would have led to its evoking less responding than $\mathrm{Y}$ would during the test.

Second, an alternative account of the effect of compound training can be given in terms of the stimulus generalization decrement that is to be expected between the end of training and the test (see St. Claire-Smith \& Mackintosh, 1974). Compound training of a previously reinforced stimulus might lead to an increased detection of a stimulus change from the end of training to testing, producing a decrement in performance to $X$. It may not be that $\mathrm{X}$ was trained in compound with a previously reinforced A, but simply that it was trained in compound, that led to its reduced performance in the test. This possibility does not uniquely apply to Experiment 1 ; it could be applied to any of the previously reported experiments in which overexpectation has been examined.

\section{EXPERIMENT 2}

Experiment 2 was an attempt to control for both the inhibition of delay and the generalization decrement accounts for the results of Experiment 1. The design of Experiment 2 is presented in Table 1 . During initial conditioning, three elements ( $\mathrm{A}, \mathrm{X}$, and $\mathrm{Y}$ ) were independently reinforced, and a fourth element (B) was nonreinforced. Both $X$ and $Y$ subsequently were reinforced in compound, one with the excitatory $A$ and one with the more neutral $\mathrm{B}(\mathrm{AX}+$ and $\mathrm{BY}+$, respectively). It was anticipated that, as a result of this treatment, $X$ would evoke a smaller response during the test. Because both $\mathrm{X}$ and $\mathrm{Y}$ were presented and reinforced an equal number of times throughout the experiment, it seems unlikely that they would differ in inhibition of delay. In addition, both $\mathrm{X}$ and $\mathrm{Y}$ experienced a shift from compound presentations to element presentations in the test, so any generalization decrement should be similar in the two.

\section{Method}

Subjects. The subjects were 16 experimentally naive SpragueDawley rats, approximately 90 days old. They were maintained as in Experiment 1 .

Apparatus. The apparatus was identical to that of Experiment 1. Two additional stimuli were used: a $76-78 \mathrm{~dB}$ clicker that produced approximately 5.5 clicks/sec and a $6-\mathrm{W}$ flashing light that flashed at a rate of $1 / \mathrm{sec}$. This light was mounted on the ceiling of the chamber, in a location that differed from that of the diffuse light.

Procedure. On the initial day of the experiment, subjects were magazine trained as in Experiment 1. Element training began on the next day. Sessions $1-4$ trained a discrimination between $\mathrm{A}+$ and $B-$, the identities of which were counterbalanced between the flashing and the steady lights. Each session consisted of 12 presentations of each stimulus, separated by an ITI that was variable around a mean of $120 \mathrm{sec}$ (the ITI duration used throughout this experiment).

Element training continued during Sessions 5-12. During Sessions 5-11, the animals received eight trials each of $\mathrm{A}+, \mathrm{B}-, \mathrm{X}+$, and $\mathrm{Y}+$ intermixed. The $\mathrm{X}$ and $\mathrm{Y}$ stimuli were counterbalanced between the clicker and the noise.

Prior to Session 12, the subjects were matched to equate response rates to $\mathrm{X}$ and $\mathrm{Y}$, while ensuring that the identities of $\mathrm{X}$ and $\mathrm{Y}$ were distributed equally between the clicker and the noise. During the first 


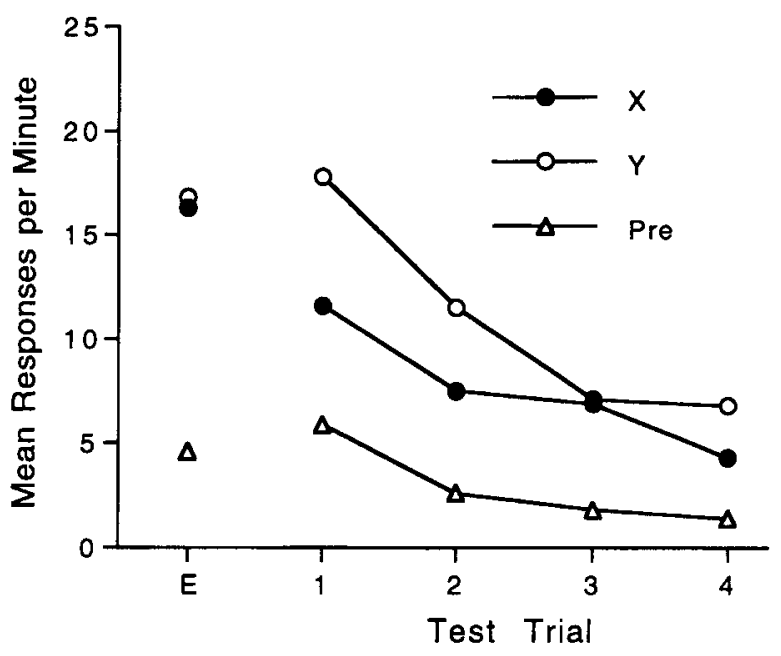

Figure 2. The results of element training and testing in Experiment 2. The mean response rates are shown during the final four trials of element training $(E)$ and during each test trial for $X, Y$, and the pre period.

half of this session, the animals received 4 trials each of $\mathrm{A}+, \mathrm{B}-$, $\mathrm{X}+$, and $\mathrm{Y}^{+}$. The trial sequence ensured that the positions of $\mathrm{X}$ and $Y$ were counterbalanced among animals. During the second half of this session, the animals received 8 trials each of $\mathrm{AX}+$ and $\mathrm{BY}+$. During Session 13, the animals received 16 trials each of $\mathrm{AX}+$ and $\mathrm{BY}+$. This training continued into the first half of Session 14, with the animals receiving 8 additional trials each of $\mathrm{AX}+$ and $\mathrm{BY}+$ Testing occurred in the second half of Session 14, with the animals receiving 8 nonreinforced test trials each of $X$ and $Y$. For half of the animals, the test trial sequence was XYYXXYYXYXYXXY; for the other half, it was YXXYYXXYXYXYYX.

\section{Results and Discussion}

The acquisition of responding during $\mathrm{A}, \mathrm{X}$, and $\mathrm{Y}$ proceeded smoothly. During the last four trials of element training, the mean levels of responding during the pre periods and during $\mathrm{A}, \mathrm{B}, \mathrm{X}$, and $\mathrm{Y}$ were 4.7, 17.2, 4.1, 16.3, and $16.8 \mathrm{rpm}$, respectively.

As in Experiment 1, there was mixed evidence for summation during the initial trials of compound training. The mean response rate during the pre periods and during $\mathrm{AX}$ and $\mathrm{BY}$ were $4.1,19.5$, and $13.9 \mathrm{rpm}$, respectively, during the first four trials. The $\mathrm{AX}$ compound produced reliably greater elevation than did the BY compound during these trials $[T(15)=15.5, p<.01]$. In addition, the elevation produced by $\mathrm{AX}$ on these trials was reliably greater than the mean of that produced on the last four trials of A and X $[T(16)=29.5, p<.05]$. However, comparing this $\mathrm{AX}$ score with the higher of the two elements for each animal did not reveal a statistically significant difference.

An examination of the mean response rates during the final trials of $Y$ and the initial trials of BY suggests that there was a small decremental effect of compounding $B$ with $\mathrm{Y}$, but this difference was neither reliable $[T(13)=$ $44]$ nor evident in the medians (14.0 and 14.5 during $Y$ and $\mathrm{BY}$, respectively). The median response rates over the same period for $A, X$, and $A X$ were 17.5, 16.0, and 19.0, respectively, which were similar to the mean rates during those stimuli.

Over the course of compound training, responding during the two compounds converged on a common level. During the final two trials of compound training, the mean response rates during the pre period and during $\mathrm{AX}$ and BY were $5.2,19.3$, and $19.1 \mathrm{rpm}$, respectively. The difference between the elevation produced by $\mathrm{AX}$ and that produced by BY was not reliable.

Figure 2 shows that test responding was greater during $Y$ than during $X$ in Experiment 2. The elevation produced by $\mathrm{Y}$ was reliably greater than that produced by $\mathrm{X}$ during the first two trials $[T(15)=19.5, p<.02]$. The change from training to the first two trials of testing was reliable for $\mathrm{X}[T(16)=14, p<.01]$ but not for $\mathrm{Y}$. In addition, this change was larger in $\mathrm{X}$ than in $\mathrm{Y}[T(16)=17.5, p<.01]$.

This experiment replicated the overexpectation effect produced in Experiment 1. The continued presentation of both $\mathrm{X}$ and $\mathrm{Y}$ during compound training ensured that these results cannot be explained by X's acquiring more inhibition of delay than did $Y$. In addition, compounding each stimulus with another ensured that the results were not due to a simple generalization decrement that might occur after any reinforced stimulus is compounded with a novel stimulus. That the test decrement was only evident in $\mathrm{X}$ suggests that reinforcing two previously reinforced stimuli in compound is critical for producing this result. Thus, it seems clear that overexpectation can be demonstrated in an appetitive Pavlovian preparation.

\section{EXPERIMENT 3}

Since the introduction of the Rescorla-Wagner model, its predictions have been tested in instrumental learning paradigms (e.g., Neely \& Wagner, 1974; Rescorla, 1973). One area in which it has been quite successful is summation (e.g., Weiss, 1972). In these experiments, responding was greater to a compound of two discriminative stimuli than to the elements. If, as the Rescorla-Wagner model asserts, overexpectation is an inevitable consequence of reinforcing such a compound, overexpectation also should appear in instrumental settings.

A procedural advantage to using an instrumental preparation is that this type of training commonly employs schedules that reinforce responding on an intermittent basis. A result of this is that, when tested in extinction, behavior is resistant to extinction. This might allow the overexpectation effect to persist for more trials than it did in the continuously reinforced Pavlovian designs used in Experiments 1 and 2.

The conceptual design of Experiment 3 was identical to that of Experiment 2, as Table 1 shows. The only difference was that, instead of being contingent on the termination of the stimulus, the reinforcer was contingent on a leverpress response during the stimulus. 


\section{Method}

Subjects. The subjects were 16 experimentally naive SpragueDawley rats, approximately 90 days old. They were maintained as in Experiments 1 and 2.

Apparatus. The apparatus was identical to that of Experiments $l$ and 2, except that the metal shield covering the lever was removed during most of the experiment. The lever measured $40 \mathrm{~mm}$ wide $\times$ $15 \mathrm{~mm}$ high and protruded $20 \mathrm{~mm}$ from the chamber wall. It was located $90 \mathrm{~mm}$ above the chamber floor.

Procedure. On the initial day, the lever was inaccessible, and the subjects were magazine trained as in Experiments 1 and 2. On the next day, the lever was accessible, and the animals received training in which each leverpress was reinforced by the delivery of a food pellet. This session ended when the animal accrued approximately 25 reinforcers. The next 2 days consisted of training with a variable interval (VI) 60 -sec schedule of reinforcement. These sessions lasted $20 \mathrm{~min}$

Element training began with the next session. During Sessions $1-4$, the animals were trained to discriminate $\mathrm{A}+$ and $\mathrm{B}-$, the identities of which were counterbalanced between the flashing and the steady lights. Responding was reinforced according to a VI 30-sec schedule during A but not during $B$. Each session consisted of 16 trials of each stimulus. The ITI was increased from $30 \mathrm{sec}$ during Session 1 to $60 \mathrm{sec}$ during Session 2 and to $90 \mathrm{sec}$ during Sessions 3 and 4. The ITI was $90 \mathrm{sec}$ from this point forward in the experiment.

During Sessions 5-11, the animals received additional element training with eight trials each of $\mathrm{A}+, \mathrm{X}+$, and $\mathrm{Y}+$, during which responding was reinforced according to a VI 30 -sec schedule, and eight trials of $\mathrm{B}-$, during which responding was not reinforced. The VI timer continued, regardless of whether a scheduled reinforcer was obtained. Any scheduled reinforcer that was not obtained by the end of the stimulus was lost. The stimulus identities, durations, and counterbalancing were identical to those of Experiment 2.

Prior to Session 12, the animals were matched to equate response rates during $\mathrm{X}$ and $\mathrm{Y}$, as in the previous experiments, again ensuring that the identities of $X$ and $Y$ were distributed equally between the clicker and the noise. During the first half of Session 12, the animals received 4 trials each of $\mathrm{A}+, \mathrm{B}-, \mathrm{X}+$, and $\mathrm{Y}+$. The second half of Session 12 consisted of 8 trials each of $\mathrm{AX}+$ and $\mathrm{BY}+$. Compound training continued during Session 13, which consisted of 16 trials each of $\mathrm{AX}+$ and $\mathrm{BY}+$. Session 14 began with 8 additional trials each of $\mathrm{AX}+$ and $\mathrm{BY}+$, followed by 8 nonreinforced test trials each of $\mathrm{X}$ and $\mathrm{Y}$. The test trial sequence for Session 14 was identi$\mathrm{cal}$ to that in Experiment 2. Sessions 15 and 16 consisted of 8 additional nonreinforced test trials of $\mathrm{X}$ and $\mathrm{Y}$. The ITI that preceded the first trial in Sessions 15 and 16 was $4 \mathrm{~min}$, in order to ensure that the pre rates would be low on the first test trial.

Two subjects failed to earn at least $25 \%$ of the reinforcers during one compound. Because this meant that they were not exposed to the response-reinforcer contingencies, they were deleted from the analysis.

\section{Resuits and Discussion}

The animals learned to press the lever during $\mathrm{A}, \mathrm{X}$, and $\mathrm{Y}$ but responded little during $\mathrm{B}$. During the last four trials of element training, the mean response rates during the pre periods and during $\mathrm{A}, \mathrm{B}, \mathrm{X}$, and $\mathrm{Y}$ were $2.6,23.8$, $5.8,21.6$, and $21.7 \mathrm{rpm}$, respectively.

The initial trials of compound training revealed robust summation. During the first four trials, the mean pre, $\mathrm{AX}$, and $\mathrm{BY}$ response rates were $1.1,26.9$, and $18.0 \mathrm{rpm}$, respectively. Greater elevation was evident in $\mathrm{AX}$ than in BY during these compound trials $[T(14)=6, p<.01]$. In addition, the elevation produced by $\mathrm{AX}$ was larger than the mean of that produced by the last four trials of $\mathrm{A}$ and $\mathrm{X}[T(13)=0, p<.001]$, as well as being larger than that produced by the larger of the elements for each ani$\operatorname{mal}[T(13)=10.5, p<.02]$.

As in Experiment 2, B seemed to exert some decremental effect on $Y$, but there was no reliable difference between the elevation produced by the first four compound trials of BY and that produced by the last four element trials of $Y[T(12)=29]$. The median response rates during Y and BY were 19.5 and 17.0, respectively.

Over the course of compound training, the levels of responding produced by AX and BY converged. During the final two trials of compound training, the mean response rates during the pre period and during $\mathrm{AX}$ and $\mathrm{BY}$ were $2.4,25.7$, and $23.7 \mathrm{rpm}$, respectively. The difference in elevation produced by $\mathrm{AX}$ and $\mathrm{BY}$ on these trials was not reliable.

As is shown in Figure 3, Y consistently evoked greater responding than did $\mathrm{X}$ throughout the three test sessions in Experiment 3. Responding extinguished during each test session but recovered at the beginning of the next session. The difference between the elevation produced by $\mathrm{X}$ and that produced by $\mathrm{Y}$, collapsed across the three test sessions, was reliable $[T(14)=15.5, p<.02]$. There was no reliable change in either $\mathrm{X}$ or $\mathrm{Y}$ from training to the first two trials of testing.

The most important observation in this experiment is that of overexpectation in instrumental conditioning. Because all prior evidence for overexpectation had been gathered in Pavlovian paradigms, this observation extends the generality of the phenomenon to another example of associative learning.

It is worth noting, however, that procedures like those in Experiments 1 and 2 also have occasionally been characterized as instrumental in nature (see Farwell \& Ayres, 1979). Although no explicit instrumental contingency was arranged between the response and the reinforcer, the animal did have to approach the magazine in order to collect the delivered reinforcer. Thus, it might be that each of our experiments studied instrumental behavior, but only Experiment 3 scheduled an explicit response-reinforcer contingency. This paper was not designed to resolve this issue (which may, in fact, be unresolvable), but we can assert that overexpectation is observable under conditions with weak and with strong response-reinforcer contingencies (see also Delamater, 1996; Rescorla, 1994).

\section{GENERAL DISCUSSION}

In these three experiments, evidence was found for overexpectation with appetitive reinforcers in both Pavlovian and instrumental conditioning. Earlier demonstrations had been confined to between-subjects comparisons and to aversive USs. Thus, these within-subjects results extend the range of situations in which the theoretically important overexpectation result has been observed.

These experiments also provided controls that enabled us to eliminate some other accounts of the overexpectation effect. Each experiment eliminated a US habituation ac- 


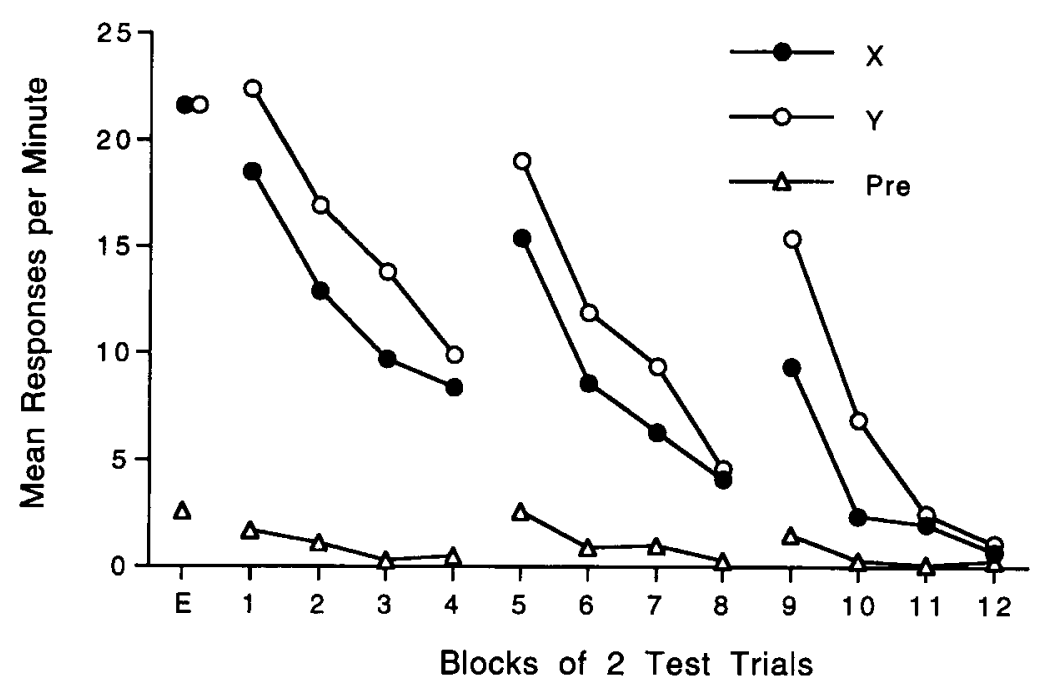

Figure 3. The results of element training and testing in Experiment 3 . The mean response rates are shown during the final four trials of element training $(E)$ and during each of the three test sessions for $X, Y$, and the pre period.

count, because any effect of US habituation should have been equal for both stimuli in our within-subjects designs. Experiment 2 eliminated inhibition of delay as a contributor to the effect by presenting $X$ and $Y$ an equal number of times. Finally, by presenting both $\mathrm{X}$ and $\mathrm{Y}$ in compound with other stimuli, Experiments 2 and 3 eliminated the hypothesis that the simple presentation of a reinforced stimulus in compound inevitably leads to a test decrement, regardless of the strength of the other stimulus in the compound. Thus, it appears that our experiments provide solid evidence for an overexpectation effect that depends on the associative strength of the compounded stimuli.

It should be noted that our experiments are not the first to attempt to demonstrate overexpectation with appetitive reinforcers and within-subjects designs. In an autoshaping experiment, Khallad and Moore (1996) found that element reinforcement followed by compound reinforcement generally produced decreased levels of responding to the elements during a test, as compared with their levels before compound training. This type of comparison is difficult to interpret, however, because it confounds training conditions with time, which makes it difficult to determine whether the observed changes from training to testing depended on the intervening compound treatment. They did show that, when compound training consisted of a US that was twice as large as that of element training, there was no test decrement (cf. Levitan, 1975). It is impossible to know, however, whether the extra reinforcers during compound training combatted a general response decrement that might have occurred with time. Because the critical comparisons in the present experiments took place in the same test session, general changes that occur with time were less likely to have contributed to the results.

In another autoshaping experiment, Rescorla (1989, Experiment 2) failed to find overexpectation. He used a design that was similar to the one in Experiment 1 reported here, but found no test difference between $X$, which received the compound treatment, and $Y$, which did not. One factor that complicates a clear interpretation of either of the recent autoshaping studies is that summation is difficult to observe in autoshaping. Because overexpectation requires summation at some level, Rescorla's failure to observe overexpectation might have been due to similar problems faced in these summation studies (see Aydin \& Pearce, 1995, 1997; Rescorla \& Coldwell, 1995). Although the autoshaping issues have yet to be resolved, the present experiments provide clear evidence that overexpectation can be demonstrated in other appetitive preparations.

These experiments were directed at overexpectation, but they incidentally provided the opportunity to observe summation during the initial trials of compound training. Experiment 3 provided strong evidence for summation in instrumental learning, but Experiments 1 and 2 provided somewhat mixed evidence for summation in magazine approach conditioning. In part, the difficulty in revealing summation in these experiments may have been due to our adopting procedures that would optimize the possibility of observing overexpectation. Thus, we did extensive training of our elements in order to guarantee substantial asymptotic responding. This may have produced a ceiling effect, which would have masked any summation. In addition, we adopted the unusual practice of presenting all of the element trials before presenting all of the compound trials. Most tests of summation intermix the stimulus presentations, allowing concurrent comparisons of compound and element performance (see Rescorla, 1997). One consequence of our not testing in this manner is that the pre rates in Experiment 1 differed between the end of element training and the beginning of compound training. This can be attributed to all of the element trials' having occurred in the first half of the session, when the local pre rates generally were higher, and to all 
of the compound trials' having occurred in the second half of the session, when the local pre rates generally were lower. This complicates the interpretation of the summation data even further, because a comparison must be made between two stimuli with different pre rates. Although we are cautious in interpreting our data as being indicative of summation, it should be noted that summation has been reported in magazine approach conditioning (e.g., by Rescorla, 1997).

The demonstrations of overexpectation reported here conform to the predictions of the Rescorla-Wagner model. According to this model, a given US can only support a fixed amount of associative strength $(\lambda)$. Reinforcing two stimuli independently causes them each to gain an associative strength of $\lambda$. When presented together in a compound, each of these stimuli will generalize $100 \%$ of its associative strength to the compound, resulting in the compound's total associative strength exceeding $\lambda$. Consequently, reinforcing the compound with the original US is said to result in the animal overexpecting the US. Continued reinforcement of the compound results in the component stimuli's losing associative strength until their sum equals $\lambda$.

A key feature in the Rescorla-Wagner model that allows it correctly to predict our results is the assumption that changes in associative strength occur as a function of the discrepancy between the current associative strength of the stimuli and the associative strength that the outcome supports. This rule has been incorporated into many modern connectionist models as the so-called delta rule (e.g., Gluck \& Bower, 1988; Kehoe, 1988; Shanks, 1991). Models that rely on this rule generally should be expected to predict overexpectation.

One model that relies on the delta rule but does not readily predict overexpectation, however, is Pearce's (1987, 1994) stimulus generalization model. Whereas the Rescorla-Wagner model argues that learning occurs to each element present on a conditioning trial, the Pearce model argues that learning occurs to the stimulus complex as a configural unit. When parts of this unit are tested alone or combined with other stimuli, the amount of responding in that test situation depends on the similarity of the trained and the tested configurations. A stimulus compound composed of two previously reinforced elements will receive generalization from each of the component elements. If each element is equally similar to the compound, each will generalize exactly $50 \%$ of its own associative strength to the compound. Consequently, the generalized excitation of the compound will equal $\lambda$, resulting in no summation effect and no discrepancy between predicted and obtained reinforcers when the compound is reinforced. This reinforcement will therefore have no effect on the associative strength of the compound or its component elements. Thus, in its simplest form, the Pearce model anticipates that summation and overexpectation will not occur, because of incomplete generalization from the elements to the compound.

It is possible, however, for the Pearce model to predict summation and overexpectation, if it additionally assumes that the context of conditioning is salient (see Pearce, 1987, pp. 66-67; Rescorla, 1997). Unfortunately, the conditions that lead to a salient context are not easy to specify. Darby and Pearce (1995) argued that the contextual stimuli present throughout a conditioning session do not enter into configural representations with the local stimuli, and that, as such, the context should not be treated by the model as a salient stimulus. Indeed, if the model assumes that the context is salient in the normal course of conditioning, it loses its ability to make many of the predictions that separate it from elemental models (e.g., Nakajima, 1997; Pearce \& Redhead, 1993, 1995; Wilson \& Pearce, 1992). As the background saliency approaches and surpasses that of the local stimuli, the Pearce model's correct predictions for many of these previous experiments become reversed. Rescorla (1997) found that the assumption that the background is more salient than the local stimuli is necessary for the Pearce model to predict summation of partially and continuously reinforced stimuli. Such an assumption, however, leads the model to predict incorrectly that extinguishing a continuously reinforced stimulus results in a partially reinforced stimulus also being extinguished. Thus, it is clear that making the assumption that allows the model to account for overexpectation could lead it to some awkward predictions.

Although it fails to predict the basic overexpectation finding of Experiment 1, the Pearce model does predict the test differences found in Experiments 2 and 3 for reasons other than overexpectation. In these experiments, it predicts $Y$ to evoke greater test responding, compared with $\mathrm{X}$, because of a process that is similar to superconditioning. After the first phase of conditioning, A, $X$, and $Y$ all should have associative strengths of $\lambda$, and $B$ should have no associative strength. The initial trials of compound conditioning should lead to a decrement in responding to BY, because the compound receives generalization from both $B$ and $Y$, resulting in an overall level of generalized excitation that is below $\lambda$. This decrement means that subsequent reinforcement of BY will lead to the BY compound's gaining its own associative strength. Conversely, when $\mathrm{A}$ is compounded with $\mathrm{X}$, there will be no decrement, because $A$ and $X$ each contribute half of their strengths $(0.5 \lambda)$ to the compound, resulting in a compound whose generalized excitatory strength is equal to $1 \lambda$. Thus, reinforcement of $\mathrm{AX}$ will not lead to the $\mathrm{AX}$ compound's gaining its own associative strength (all of its strength will be generalized from $A$ and $X$ ). As a result of its receiving generalized excitation from BY, stimulus $Y$ will evoke greater responding at the test than it did before compound training, but, because stimulus $\mathrm{X}$ receives no generalized excitation from $\mathrm{AX}$, it will evoke a level of responding that is similar to that evoked before compound training. Thus, the Pearce model predicts that the $\mathrm{AX}$ and $\mathrm{BY}$ compound treatment will produce an increment in responding during $\mathrm{Y}$ but no change in responding during $X$.

There are, however, several reasons to question this analysis. First, in another magazine approach experiment, Pearce and Redhead (1995) found that simple nonrein- 
forcement of a stimulus (B) does not cause superconditioning of a reinforced stimulus (Y) when the two stimuli are reinforced in compound. Instead, they found that superconditioning occurs only when B is given explicit inhibitory treatment before being compounded with $\mathrm{Y}$. Second, the decrement that $\mathrm{B}$ produced in $\mathrm{Y}$ in Experiments 2 and 3 reported here was marginal, which suggests that the $\mathrm{A}+, \mathrm{B}-$ treatment in our experiments led to little inhibition in $\mathrm{B}$ and that combining $\mathrm{B}$ with $\mathrm{Y}$ did not lead to substantial generalization decrement, either through conditioned inhibition or through external inhibition, as predicted by the Pearce model. Indeed, this result is similar to that of recent experiments by Aydin and Pearce (1995) and by Rescorla (1997), both of which yielded no evidence for differential inhibition after an $\mathrm{A}+, \mathrm{B}$ - discrimination; that is, when $\mathrm{A}$ and $\mathrm{B}$ were presented in compound, there was no decrement in $A B$ trials as compared with $\mathrm{A}+$ trials. Third, instead of being due to an increase in responding during $\mathrm{Y}$, as the Pearce model predicts, the $X-Y$ test difference appears to have been due to a decrement in $X$, relative to the end of element training. However, it would be necessary to include additional controls for the possibility of general response changes that occur with time in order to demonstrate clearly that the $\mathrm{X}-\mathrm{Y}$ test difference was due to a decrement in the strength of $X$.

Even if superconditioning did contribute to the $\mathrm{X}-\mathrm{Y}$ differences observed in Experiments 2 and 3, it is clear that the overexpectation result in Experiment 1 cannot be due to superconditioning, because all stimuli were reinforced prior to compound training. The results of Experiment 1 therefore raise problems particularly for the Pearce model, unless it makes assumptions about context saliency, which might, in turn, limit its utility for correctly predicting the results that separate it from the Rescorla-Wagner model.

Although there are important differences between the Pearce model and the Rescorla-Wagner model, both share the fundamental assumption that the differences observed between $\mathrm{X}$ and $\mathrm{Y}$ during testing reflect differences in learning about these two stimuli. It is worth noting that these data also are consistent with models that view $\mathrm{X}$ and $\mathrm{Y}$ as having similar associative strengths that are differentially reflected in performance. Comparator theories (Gibbon \& Balsam, 1981; Miller \& Schachtman, 1985 ) would argue that the difference between $X$ and $Y$ at a test occurred because compound training embedded them in different comparator stimuli. The comparator stimulus for $\mathrm{X}$ was $\mathrm{A}$, which also was reinforced, but the comparator stimulus for $\mathrm{Y}$ was either the context alone (Experiment 1) or B (Experiments 2 and 3), neither of which ever was reinforced. These theories argue that the response will not be performed adequately if the associative strengths of the comparator stimuli are high, relative to that of the target test stimuli. Therefore, because X's comparator stimulus was of high value, its response was suppressed. By contrast, Y's comparator was of low value, which resulted in no suppression.
Recent findings from other cue-competition experiments, however, complicate this extrapolation of comparator theory. Miller and his colleagues (Denniston et al., 1996; Miller \& Matute, 1996) have argued that a biologically significant stimulus (i.e., one that has been conditioned with a US) is not subject to performance decrements that are based on the current state of its comparator stimulus. Their data come primarily from backwards-blocking paradigms, in which a compound stimulus (AX) is reinforced prior to $\mathrm{A}+$ alone trials. This type of treatment, according to comparator theory, should result in attenuated performance to $\mathrm{X}$, because its comparator stimulus, $\mathrm{A}$, is of higher associative strength after $\mathrm{A}+$ training. However, a backwards-blocking effect of this sort does not readily occur in nonhuman animals when biologically significant stimuli are used. To account for these results, Miller and colleagues have proposed modifying comparator theory in such a way that performance to biologically significant target stimuli cannot be attenuated by inflating the value of their comparator context. With such a modification, overexpectation would not be predicted because it requires a biologically significant stimulus to weaken in performance simply as a result of its being paired with another biologically significant comparator stimulus. The present demonstrations of overexpectation, therefore, suggest problems for this modified version of comparator theory. To account for the present results, the theory would have to be modified further to assume that inflating the value of a target's comparator stimulus by reassigning it to a different comparator stimulus (as occurred in the present experiments) is more effective in decreasing performance to a biologically significant target stimulus than is inflating the value of the comparator stimulus through separate reinforcement techniques (as occurs in backwards-blocking designs).

Regardless of the processes underlying overexpectation, these experiments show that it does occur with appetitive reinforcers by using designs that control for other plausible interpretations. Thus, it appears that the discrepancy between anticipated and obtained outcomes is fundamental to determining the effectiveness of reinforcement in both Pavlovian and instrumental conditioning.

\section{REFERENCES}

Aydin, A., \& PearCE, J. M. (1995). Summation in autoshaping with short- and long-duration stimuli. Quarterly Journal of Experimental Psychology, 48B, 215-234.

Aydin, A., \& Pearce, J. M. (1997). Some determinants of response summation. Animal Learning \& Behavior, 25, 108-121.

Bourne, M. J. (1990). Compound conditioning of separately pretrained conditioned stimuli evoking dissimilar conditioned responses. Bulletin of the Psychonomic Society, 28, 451-454.

Chapman, G. B., \& Robbins, S. J. (1990). Cue interaction in human contingency judgment. Memory \& Cognition, 18, 537-545.

DARBY, R. J., \& PEARCE, J. M. (1995). Effects of context on responding during a compound stimulus. Journal of Experimental Psychology: Animal Behavior Processes, 21, 143-154.

DELAMATER, A. R. (1996). Effects of several extinction treatments upon the integrity of Pavlovian stimulus-outcome associations. Animal Learning \& Behavior, 24, 437-449. 
Denniston, J. C., Miller, R. R., \& Matute, H. (1996). Biological significance as a determinant of cue competition. Psychological Science, 7, 325-331.

Dickinson, A. (1980). Contemporary animal learning theory. New York: Cambridge University Press.

DiCKINSON, A. (1989). Expectancy theory in animal conditioning. In S. B. Klein \& R. R. Mowrer (Eds.), Contemporary learning theories: Pavlovian conditioning and the status of traditional learning theory (pp. 279-308). Hillsdale, NJ: Erlbaum.

Dickinson, A., Shanks, D., \& Evenden, J. (1984). Judgement of actoutcome contingency: The role of selective attribution. Quarterly Journal of Experimental Psychology, 36A, 29-50.

FARWELL, B. J., \& AYRES, J. B. (1979). Stimulus-reinforcer and responsereinforcer relations in the control of conditioned appetitive headpoking ("goal tracking") in rats. Learning \& Motivation, 10, 295-312.

Ganesan, R., \& PearCE, J. M. (1988). Interactions between conditioned stimuli for food and water in the rat. Quarterly Journal of Experimental Psychology, 40B, 229-241.

GibBon, J., \& Balsam, P. D. (1981). Spreading association in time. In C. M. Locurto, H. S. Terrace, \& J. Gibbon (Eds.), Autoshaping and conditioning theory (pp. 219-253). New York: Academic Press.

GLUCK, M. A., \& BOWER, G. H. (1988). From conditioning to category learning: An adaptive network model. Journal of Experimental Psychology: General, 117, 227-247.

KAMIN, L. J. (1968). "Attention-like" processes in classical conditioning. In M. R. Jones (Ed.), Miami symposium on the prediction of behav ior: Aversive stimulation (pp. 9-33). Coral Gables, FL: University of Miami Press.

KamIN, L. J. (1969). Predictability, surprise, attention, and conditioning. In B. A. Campbell \& R. M. Church (Eds.), Punishment and aversive behavior (pp. 279-296). New York: Appleton-Century-Crofts.

Kamin, L. J., \& GaIONI, S. J. (1974). Compound conditioned emotional response conditioning with differentially salient elements in rats. Journal of Comparative \& Physiological Psychology, 87, 591-597.

KEHOE, E. J. (1986). Summation and configuration in conditioning of the rabbit's nictitating membrane response to compound stimuli. Journal of Experimental Psychology: Animal Behavior Processes, 12, 186-195.

KенOE, E. J. (1988). A layered network model of associative learning: Learning to learn and configuration. Psychological Review, 95, 411-433.

KhallaD, Y., \& MoORE, J. (1996). Blocking, unblocking, and overexpectation in autoshaping with pigeons. Journal of the Experimental Analysis of Behavior, 65, 575-591.

KREMER, E. F. (1978). The Rescorla-Wagner model: Losses in associative strength in compound conditioned stimuli. Journal of Experimental Psychology: Animal Behavior Processes, 4, 22-36.

LEviTAN, L. (1975). Tests of the Rescorla-Wagner model of Pavlovian conditioning. Bulletin of the Psychonomic Society, 6, 265-268.

Miller, R. R., Hallam, S. C., \& Grahame, N. J. (1990). Inflation of comparator stimuli following CS training. Animal Learning \& Behavior, 18, 434-443.

Miller, R. R., \& MATUte, H. (1996). Biological significance in forward and backward blocking: Resolution of a discrepancy between animal conditioning and human causal judgment. Journal of Experimental Psychology: General, 4, 370-386.

Miller, R. R., \& Schachtman, T. R. (1985). Conditioning context as an associative baseline: Implications for response generation and the nature of conditioned inhibition. In R. R. Miller \& N. E. Spear (Eds.), Information processing in animals: Conditioned inhibition (pp. 51-88). Hillsdale, NJ: Erlbaum.
NaKajima, S. (1997). Failure of inhibition by $B$ over $C$ after $A+, A B-$, $\mathrm{ABC}+$ training. Journal of Experimental Psychology: Animal Behavior Processes, 23, 482-490.

NeELY, J. H., \& WAGNER, A. R. (1974). Attenuation of blocking with shifts in reward: The involvement of schedule-generated contextual cues. Journal of Experimental Psychology, 102, 751-763.

Pearce, J. M. (1987). A model of stimulus generalization for Pavlovian conditioning. Psychological Review, 94, 61-73.

PearCe, J. M. (1994). Similarity and discrimination: A selective review and a connectionist model. Psychological Review, 101, 587-607.

PearCe, J. M., \& Redhead, E. S. (1993). The influence of an irrelevant stimulus on two discriminations. Journal of Experimental Psychology: Animal Behavior Processes, 19, 180-190.

Pearce, J. M., \& Redhead, E. S. (1995). Supernormal conditioning. Journal of Experimental Psychology: Animal Behavior Processes, 21, 155-165.

Rescorla, R. A. (1970). Reduction in the effectiveness of reinforcement after prior excitatory conditioning. Learning \& Motivation, 1, 372-381.

REsCORLA, R. A. (1973). Evidence for a "unique stimulus" account of configural conditioning. Journal of Comparative \& Physiological Psychology, 85, 331-338.

RESCORLA, R. A. (1989). Redundant treatment of neutral and excitatory stimuli in autoshaping. Journal of Experimental Psychology: Animal Behavior Processes, 15, 212-223.

ResCoRla, R. A. (1994). Control of instrumental performance by Pavlovian and instrumental stimuli. Journal of Experimental Psychology: Animal Behavior Processes, 20, 44-50.

RESCORLA, R. A. (1997). Summation: Assessment of a configural theory. Animal Learning \& Behavior, 25, 200-209.

REsCORLA, R. A., \& Coldwell, S. E. (1995). Summation in autoshaping. Animal Learning \& Behavior, 23, 314-326.

Rescorla, R. A., \& Wagner, A. R. (1972). A theory of Pavlovian conditioning: Variations in the effectiveness of reinforcement and nonreinforcement. In A. H. Black \& W. F. Prokasy (Eds.), Classical conditioning II: Current research and theory (pp. 64-99). New York: Appleton-Century-Crofts.

SHANKS, D. R. (1991). Categorization by a connectionist network. Journal of Experimental Psychology: Learning. Memory, \& Cognition, 17, 433-443.

St. Claire-Smith, R., \& Mackintosh, N. J. (1974). Complete suppression to a compound CS does not block further conditioning to each element. Canadian Journal of Psychology, 28, 92-101.

WAGNER, A. R. (1971). Elementary associations. In H. H. Kendler \& J. T. Spence (Eds.), Essays in neobehaviorism: A memorial volume to Kenneth W. Spence (pp. 187-213). New York: Appleton-Century-Crofts.

WAGNER, A. R. (1981). SOP: A model of automatic memory processing in animal behavior. In N. E. Spear \& R. R. Miller (Eds.), Information processing in animals: Memory mechanisms (pp. 5-47). Hillsdale, $\mathrm{NJ}$ : Erlbaum.

WEISS, S. J. (1972). Stimulus compounding in free-operant and classical conditioning: A review and analysis. Psychological Bulletin, 78, 189-208.

Williams, B. A. (1994). Blocking despite changes in reinforcer identity. Animal Learning \& Behavior, 22, 442-457.

Wilson, P. N., \& PEARCE, J. M. (1992). A configural analysis for featurenegative discrimination learning. Journal of Experimental Psychology: Animal Behuvior Processes, 18, 265-272.

(Manuscript received February 17, 1997; revision accepted for publication December 3, 1997.) 